\title{
Review Paper: \\ Challenges of Undergoing Dialysis With Emphasis on Aging Syndromes in the Elderly
}

\author{
Ehterm Sadat Ilali ${ }^{1},{ }^{*}$ Hamid Peyrovi ${ }^{2}$, Robab Sahaf $^{3}$, Ahmad Ali Akbari Kamrani ${ }^{3}$, Fatemeh Sepahbodi ${ }^{4}$
}

1. Department of Nursing, School of Nursing and Midwifery, Mazandaran University of Medical Sciences, Sari, Iran.

2. Department of Critical Care Nursing, School of Nursing and Midwifery, Iran University of Medical Sciences, Tehran, Iran.

3. Iranian Research Center on Aging, University of Social Welfare and Rehabilitation Sciences, Tehran, Iran

4. Department of Nephrology, School of Medicine, Mazandaran University of Medical Sciences, Sari, Iran.

Received: 29 Jun. 2016

Accepted: 13 Nov. 2016

Keywords:

Aged, Renal dialysis, Challenges, Elderly syndromes

\section{A B STRACT}

Objectives Dialysis is a therapeutic method suitable for elderly people with chronic renal failure. However, the patient faces with multiple challenges. This study explores the challenges of elderly people with dialysis for better identification and prevention of its side effects.

Methods \& Materials In this study, a comprehensive search of electronic databases of PubMed, Web of science, ProQuest, Scopus, Elsevier, Google scholar, and IranMedex was conducted using the key words "Aged," "Renal dialysis," "challenges," and "elderly syndromes" with no time limitation until the end of 2015.

Results Elderly people undergoing dialysis have multiple physical and mental problems. They consume more drugs and have comorbidity compared to young people. This leads to lower quality of life in them compared to other age groups. Elderly people under dialysis experience more challenges, such as aging syndromes (including falls, functional disability, frailty, and cognitive impairment) compared to healthy people. The prevalence of these disorders is more in the elderly people undergoing dialysis leading to disability and loss of independence and increased mortality rate.

Conclusion The number of elderly people undergoing dialysis has increased, and it seems that we need to know more about the problems of this age group. With the increasing awareness and knowledge about these problems, we can plan prevention, care, and rehabilitation programs for the elderly people with dialysis.

\section{* Corresponding Author:}

Hamid Peyrovi, PhD

Address: Department of Critical Care Nursing, School of Nursing and Midwifery, Iran University of Medical Sciences, Tehran, Iran.

Tel: +98 (21) 43751606

E-mail: hamidpeyravi@yahoo.com 


\section{مطالعه مرورى: خالشهاى سالمندان دياليزى با تأكيد بر سندرومهاى سالمندى}

احترامالسادات اليلالى'، "حميد بيروى '، رباب صحاف"، احمدعلى اكبرى كامرانى"، فاطمه اسيهبدى"

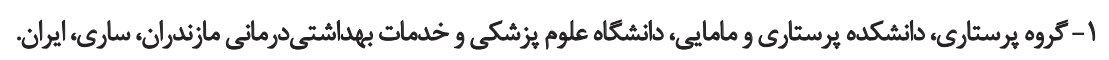

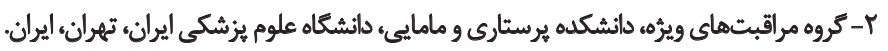

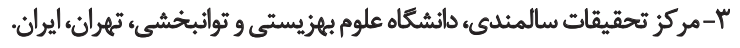

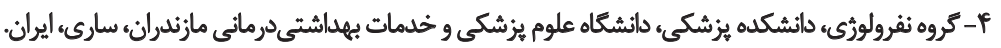

تاريخ دريافت: · تير هوسا

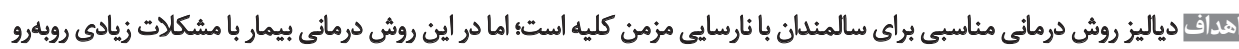

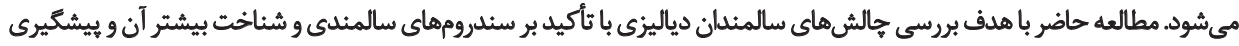

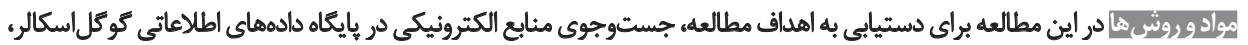

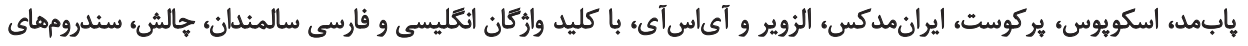

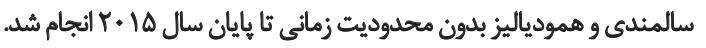

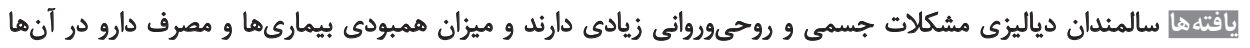

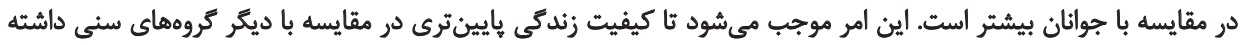

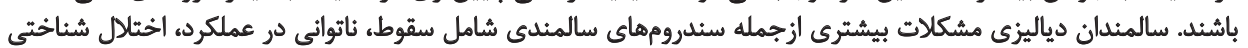

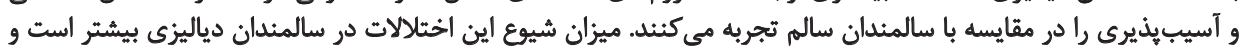

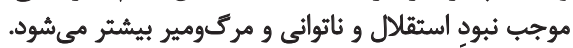

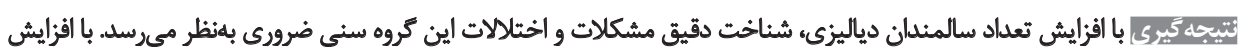

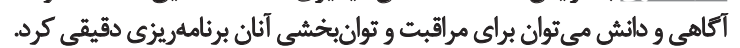

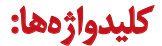

سالمندان، همودياليز،

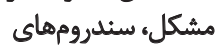

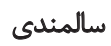

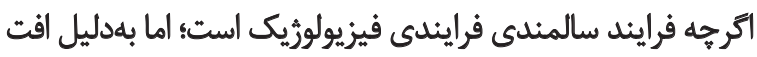

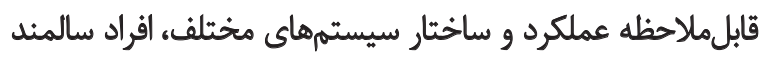

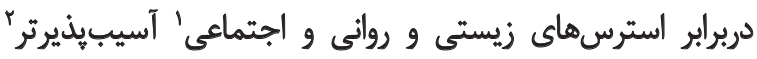

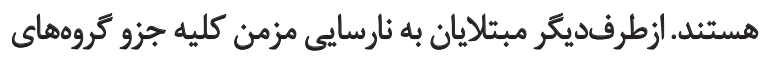

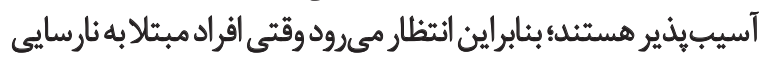

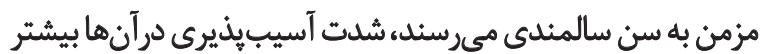

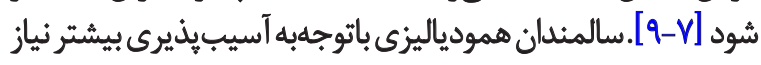

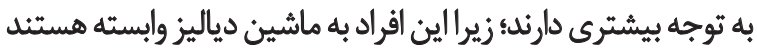

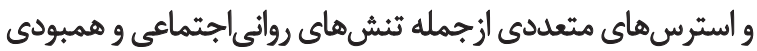

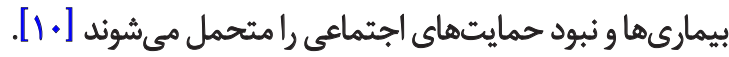
باتوجهبه افزايش تعداد سالمندان تحتدرمان با همودياليز در

1. Biopsycosocial

2. Frail

doles

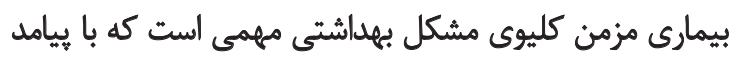

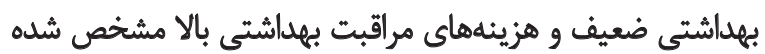

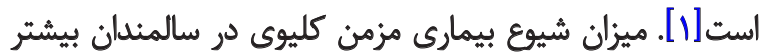

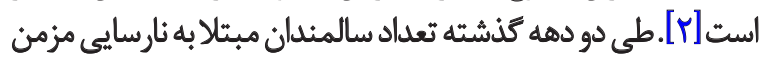

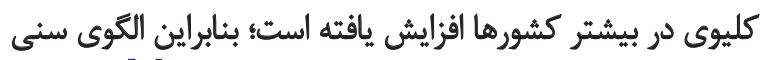

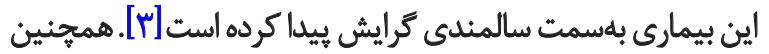

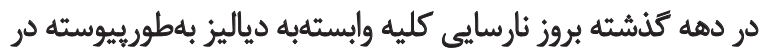

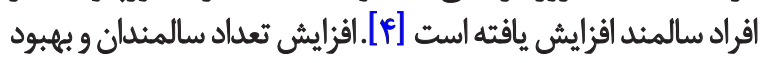

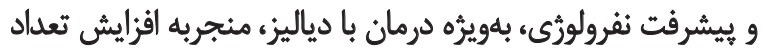

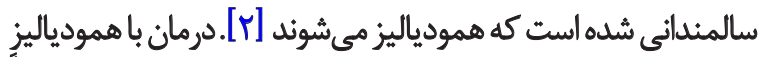

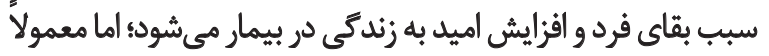

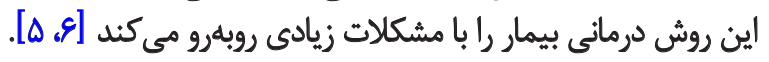

: نويسئده مسئول:

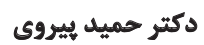

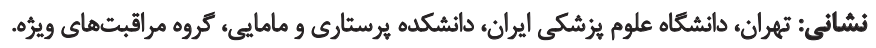

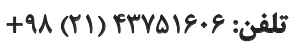
بست الكترونيكي: hamidpeyravi@yahoo.com 
آسيبذيذيرى" و اختلال شناختى كزارش شد.

هالش هاي سالمندان دياليزى

سقوط

سندروم سقوط يكى از مشكلات شايع سالمندان دياليزى

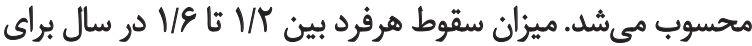

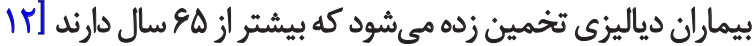

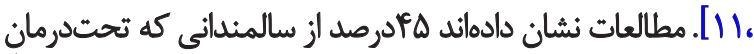

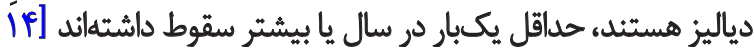

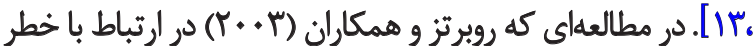

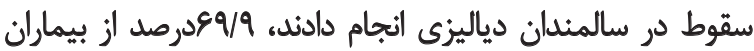

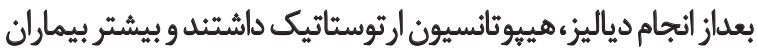

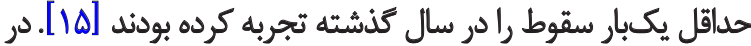

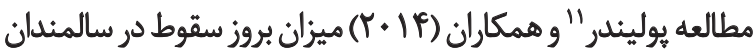

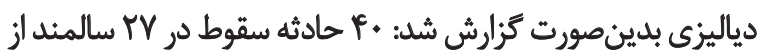

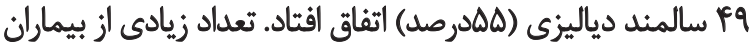
ان (Ifl)

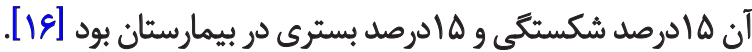

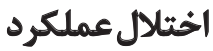

از قالشهاى ديكر سالمندان دياليزى، كاهش استقلال

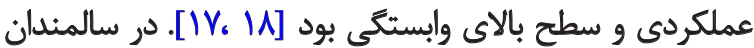

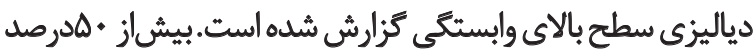

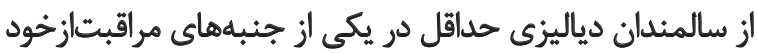

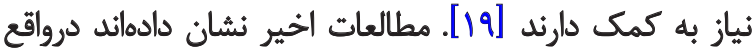

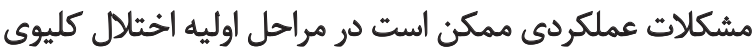

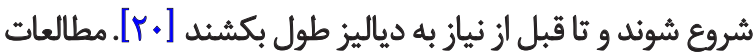

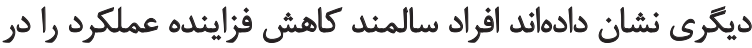

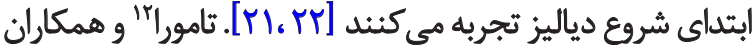

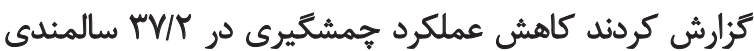

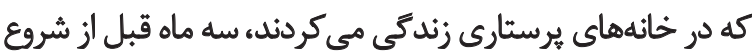

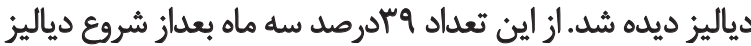

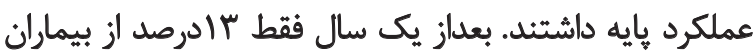

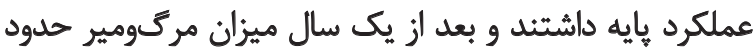

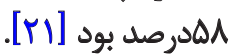

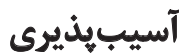

مطالعات زيادى تأكيد داشتند كه بسيارى از سالمندان دياليزى اختلال شناختى و اختلال عملكردى دارند و آسيبيذيدير هستيند.

10. Frailty

11. Poulinder

12. Tamura
كشور و اهميت مشكلات مرتبط با آن (مشكلات متعدد طبّى

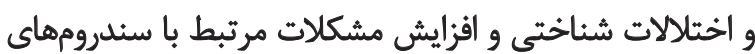

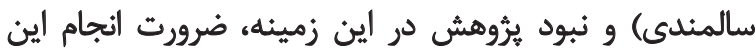

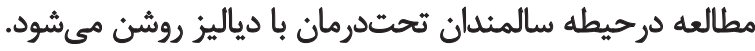

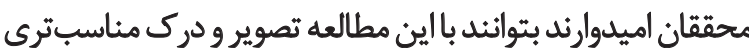
از زندكى سالمندان همودياليزى ارائه كنثد.

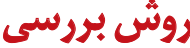

در اين مطالعه جستوجوى منابع الكترونيكى شامل تمام مقالات

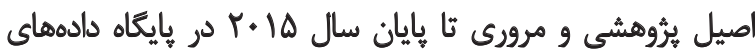

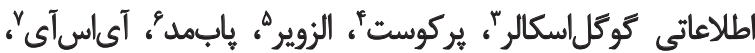

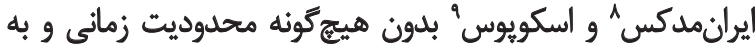

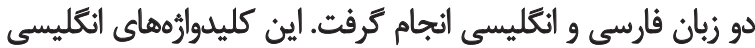

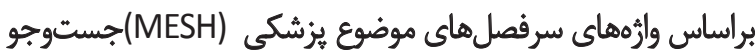
شد: challenges, Renal Dialysis, Aged, elderly syndrome.

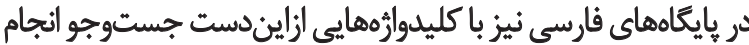

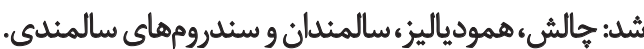

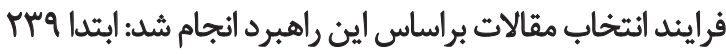

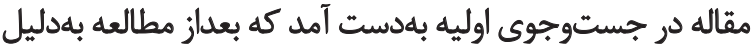

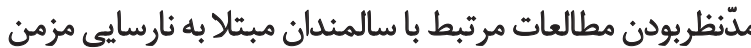

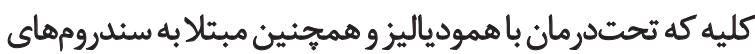

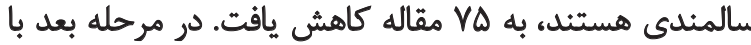

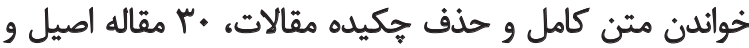
مرورى براى بررسى انتخاب شد.

معيارهاى ورود مقالات به اين مطالعه عبارت بود ازئ مدّنظر

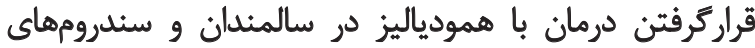

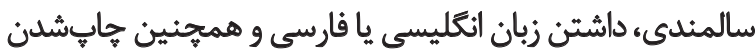

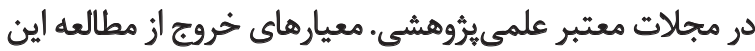

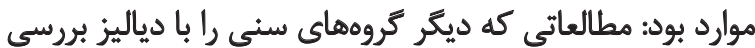
كرده بودند، جكيده مقالات، نامه به سردبير و مقالاتى به به زبانى

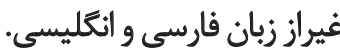

يأقتهها

نتايج اين يُروهش در جالشهاى سالمندان دياليزى با تأكيد بر سندرومهاي سالمندى شامل سقوط، اختالئ سال در عملكرد،
3. Google Scholar
4. ProQuest
5. Elsevier
6. Pubmed
7. ISI (Web of Science)
8. Iran Medex
9. Scopus 
مطالعات اخير نشان داد در سالمندانى كه دياليز ميشوند و

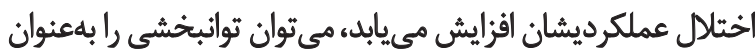

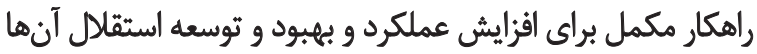

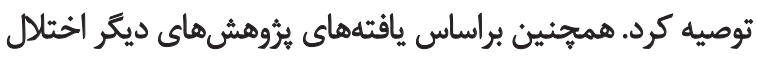
شناختى يكى ديكر از مشكلات سالمندان است كه به با انجام دياليز

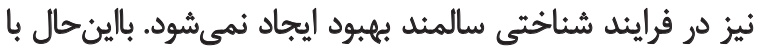

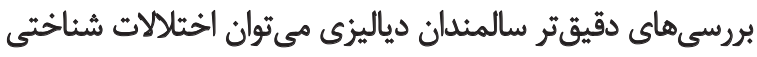

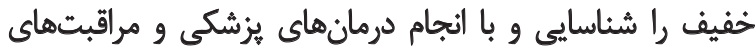

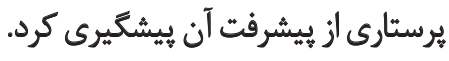

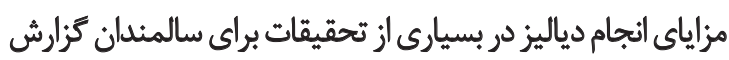

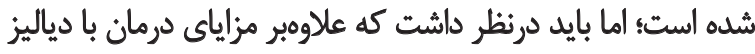

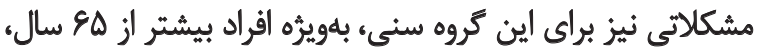

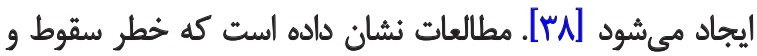

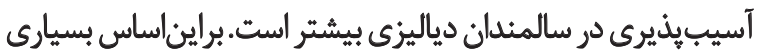

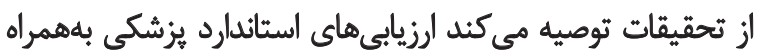

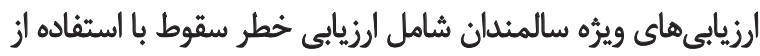

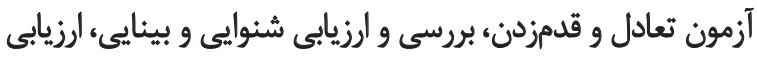

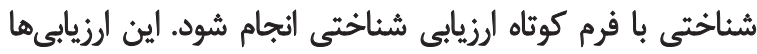

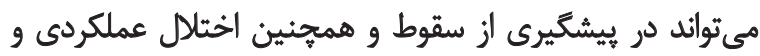

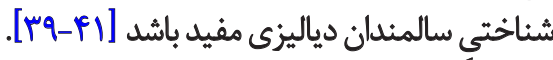

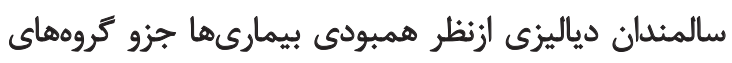

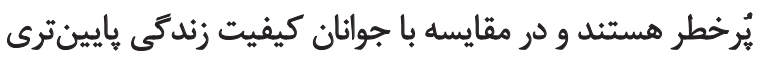

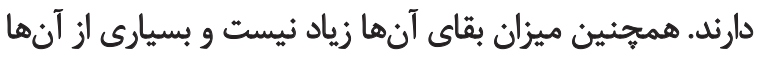

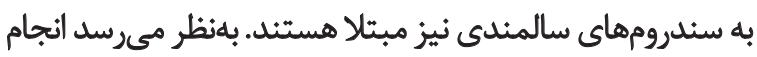

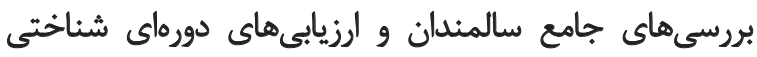

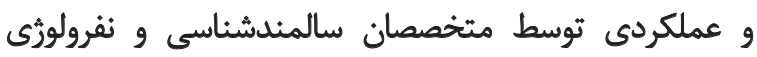

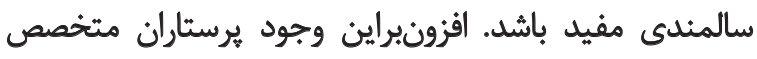

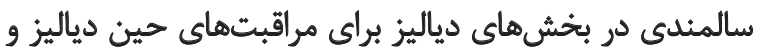

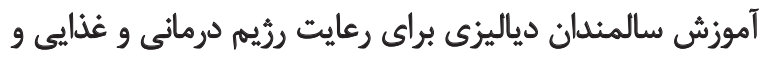

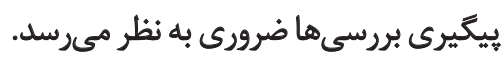

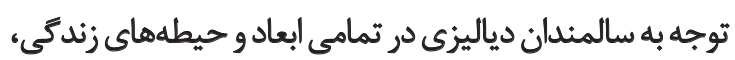

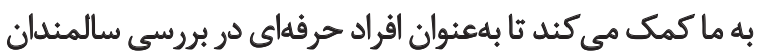

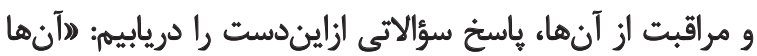

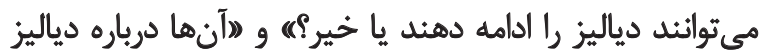

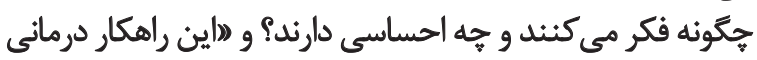
براي آنها سودمئد است؟ مكن.

\section{تتيجهيرى نمهايى}

بهدليل تغييرات سنى در جمعيت بيماران دياليزى و افزايش

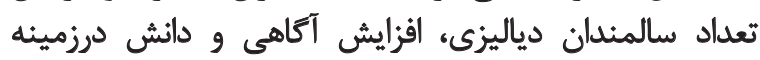

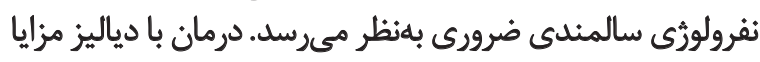

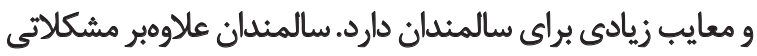

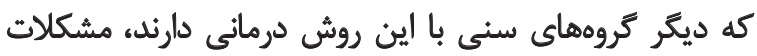

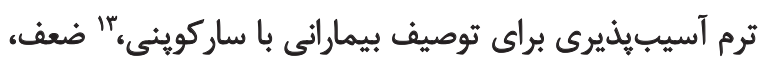

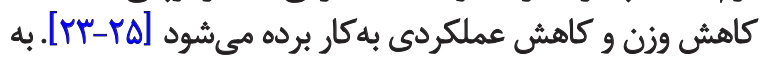

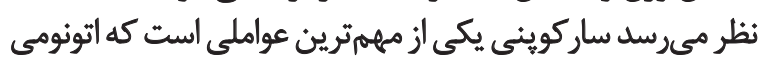

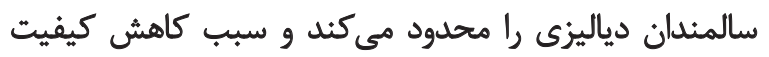

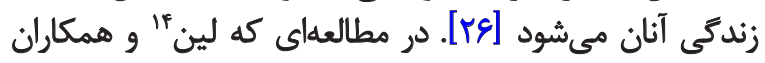

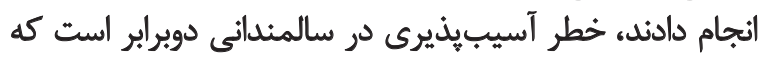

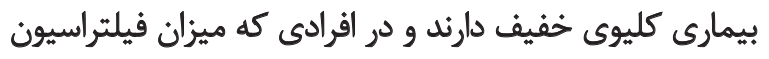

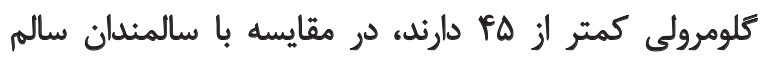

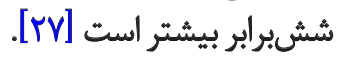

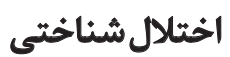

ارتباط بين اختلال شناختيى و نارسايي مزمن كليه در مطالعات إختات

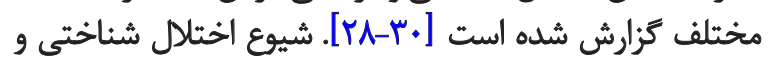

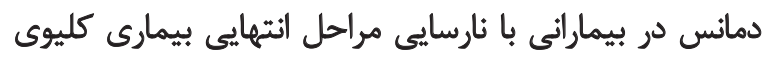

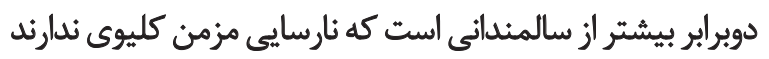

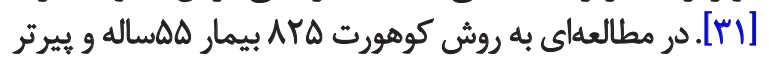

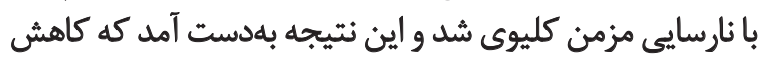

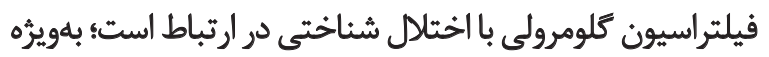

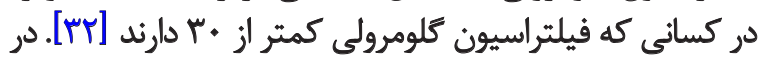

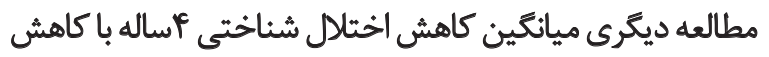

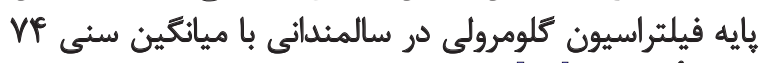

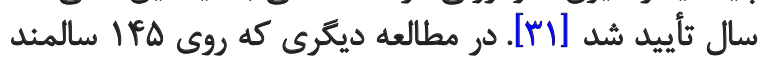

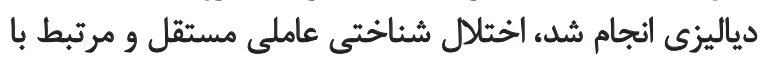

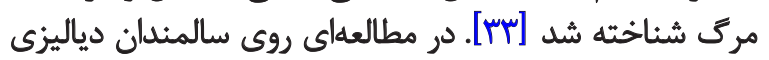

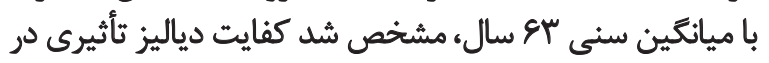

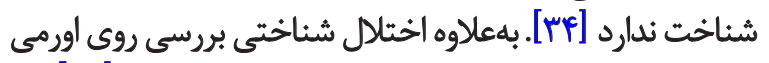

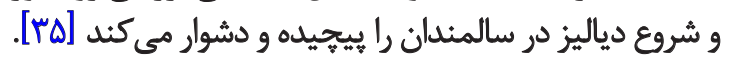

ثب در اين مطالعه جالشهاى سالمندان دياليزى با تأكيد بر

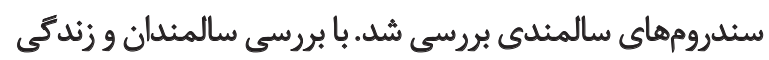

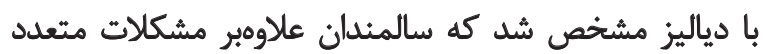

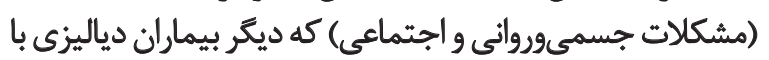

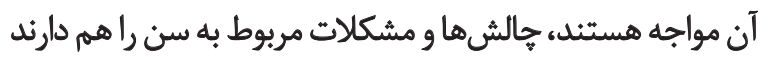

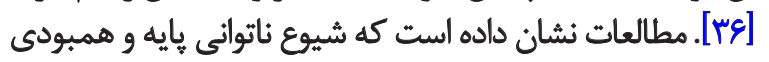

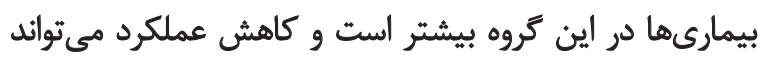

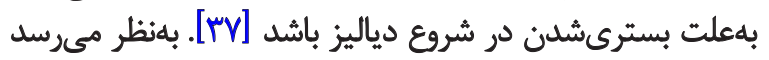

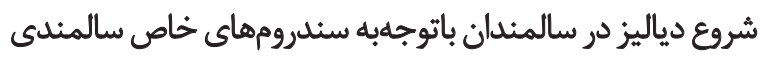

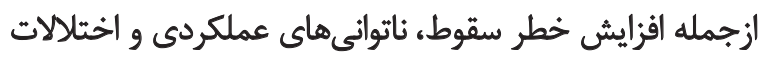

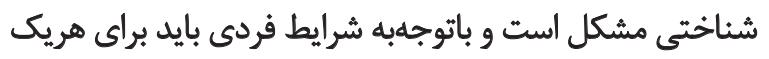

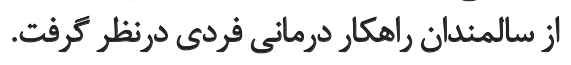

13. Sarcopenia

14. Leen 


\section{References}

[1] Couser W, Remuzzi G, Mendis S, Tonelli M. The contribution of chronic kidney disease to the global burden of major noncommunicable disease. Kidney International Journal. 2011; 80(12):1258270. doi: 10.1038/ki.2011.368

[2] Tonelli M, Riella M. Chronic kidney disease and the aging population. Iranian Journal of Kidney Disease. 2014; 4(8):87-92. doi: 10.1111/nep.12185

[3] Jager K, Dijk P, Dekker F, Stengel B, Simpson K, Briggs J. The epidemic of aging in renal replacement therapy: An update on elderly patients and their outcomes. Clinical Nephrology. 2003; 60(11):352-60. doi: 10.5414/cnp60352

[4] Kurella M, Covinsky K, Collins A, Chertow G. Octogenarians and nonagenarians starting dialysis in the United States. Annals of Internal Medicine. 2007; 146(3):177-83. doi: 10.7326/0003-4819146-3-200702060-00006

[5] Moran A, Scott P, Darbyshire P. Existential boredom: The experience of living on hemodialysis therapy. Medical Humanities. 2009; 35(2):70-75. doi: 10.1136/jmh.2009.001511

[6] Morehouse R, Colvin E, Maykut P. Nephrology nurse-patient relationships in the outpatient dialysis setting. Nephrology Nursing Journal. 2001; 28(3):295-300. PMID: 1214345

[7] Brown W. Hemodialysis in elderly patients. International Urology and Nephrology. 2000; 32(1):113-26. doi: /10.1007/978-94011-4088-1_11

[8] Winchester J, Rakowski T. End-stage renal disease and its management in older adults. Clinics in Geriatric Medicine. 1998; 14(2):255-65

[9] Dharmarajan T, Kaul N, Russell R. Dialysis in the old: A centenarian nursing home resident with end-stage renal disease. Journal of the American Medical Directors Association. 2004; 5(3):18691. doi: 10.1016/s1525-8610(04)70113-9

[10] Calvin A. Haemodialysis patients and end-of-life decisions: A theory of personal preservation. Journal of Advanced Nursing. 2004; 46(5):558-66. doi: 10.1111/j.1365-2648.2004.03030.x

[11] Desmet C, Beguin C, Swine C, Jadoul M. Falls in hemodialysis patients: Prospective study of incidence, risk factors, and complications. American Journal of Kidney Disease. 2005; 45(1):148-53. doi: 10.1053/j.ajkd.2004.09.027

[12] Cook W, Tomlinson G, Donaldson M, Markowitz S, Naglie $\mathrm{G}$, Sobolev B, et al. Falls and fall-related injuries in older dialysis patients. Clinical Journal of the American Society of Nephrology. 2006; 1(6):1197-204. doi: 10.2215/cjn.01650506

[13] Kutner N. Promoting functioning and well-being in older CKD patients: review of recent evidence. International Urology and Nephrology. 2008; 40(4):1151-158. doi: 10.1007/s11255-008-9469-x

[14] Odden M. Physical functioning in elderly persons with kidney disease. Advances in Chronic Kidney Disease. 2010; 17(4):348-57. doi: 10.1053/j.ackd.2010.02.002

[15] Roberts R, Kenny R, Brierley E. Are elderly hemodialysis patients at risk of falls and postural hypotension? International Urology and Nephrology. 2003; 35(3):415-21. doi: 10.1023/b:urol.0000 $022866.07751 .4 a$

[16] Polinder-Bos HA, Emmelot-Vonk MH, Gansevoort R, Diepenbroek A, Gaillard CA. High fall incidence and fracture rate in
خاص سنى ازجمله سندرومهاى سالمندى رانيز تجربه مى كنيند.

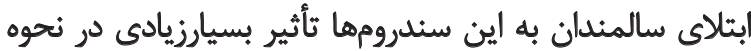

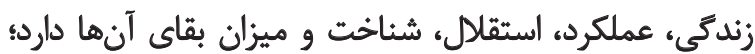

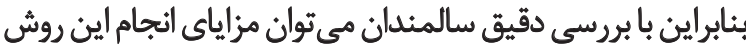

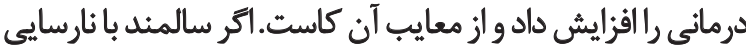

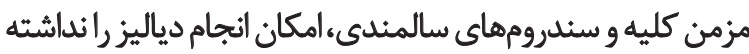

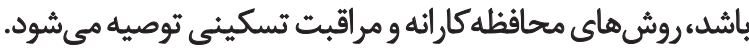

مجدوديتها

از محدوديتهاي اين مطالعه مي توان به دسترسىنداشتن به

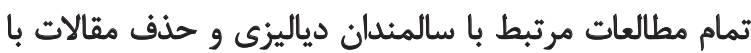

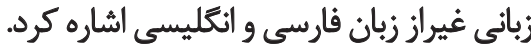

Loleinin

سالمندان مبتلا به نارسايیى مزمن كليوى به درمانهاى

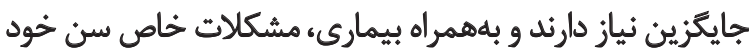

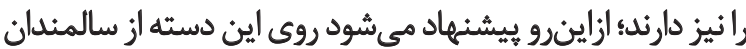

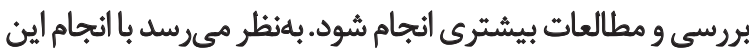

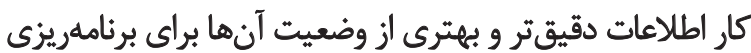

$$
\text { درماني و مراقبتى خواهيم داشت. }
$$

$$
\text { تشكر و قدرداني }
$$

مثاله حاضر، مثالهاى مرورى است و حامى مالى ندارد. 
elderly dialysis patients. Netherlands Journal of Medicine. 2014; 72(10):509-15. PMID: 26219755

[17] Ifudu O, Paul H, Homel P, Friedman E. Predictive value of functional status for mortality in patients on maintenance hemodialysis. American Journal of Nephrology. 1998; 18(2):109-16. doi: $10.1159 / 000013318$

[18] McClellan W, Anson C, Birkeli K, TuttleE. Functional status and quality of life: Predictors of early mortality among patients entering treatment for end stage renal disease. Journal of Clinical Epidemiology. 1991; 44(1):83-89. doi: 10.1016/0895-4356(91)90204-m

[19] Cook W, Jassal S. Functional dependencies among the elderly on hemodialysis. Kidney International. 2008; 73(11):1289-295. doi: 10.1038/ki.2008.62

[20] Fried L, Lee J, Shlipak M, Chertow G, Green C, Ding J, et al. Chronic kidney disease and functional limitation in older people: Health, aging and body composition study. Journal of the American Geriatrics Society. 2006; 54(5):750-56. doi: 10.1111/j.15325415.2006.00727.x

[21] Tamura MK, Covinsky K, Chertow G, Yaffe K, Landefeld C, McCulloch C. Functional status of elderly adults before and after initiation of dialysis. New England Journal of Medicine. 2009; 361(16):1539-547. doi: 10.1056/nejmoa0904655

[22] Jassal S, Chiu E, Hladunewich M. Loss of independence in patients starting dialysis at 80 years of age or older. New England Journal of Medicine. 2009; 361(16):1612-613. doi: 10.1056/ nejmc0905289

[23] Fried L, Tangen C, Walston J, Newman A, Hirsch C, Gottdiener $\mathrm{J}$, et al. Frailty in older adults: Evidence for a phenotype. Journal of Gerontology series A: Biological Sciences and Medical Sciences. 2001; 56(3):146-56. doi: 10.1093/gerona/56.3.m146

[24] Wells J, Seabrook J, Stolee P, Borrie M, Knoefel F. State of the art in geriatric rehabilitation: Part I-Review of frailty and comprehensive geriatric assessment. Archive of Physical Medicine and Rehabilitation. 2003; 84(6):890-97. doi: 10.1016/s00039993(02)04929-8

[25] Bandeen-Roche K, Xue Q, Ferrucci L, Walston J, Guralnik J, Chaves P. Phenotype of frailty: Characterization in the women's health and aging studies. Journal of Gerontology series A: Biological Sciences and Medical Sciences. 2006; 61(3):262-66. doi: 10.1093/gerona/61.3.262

[26] Domański M, Ciechanowski K. Sarcopenia: A major challenge in elderly patients with end-stage renal disease. Journal of Aging Research. 2011; 2012(2012):1-12. doi: 10.1155/2012/754739

[27] Wilhelm-Leen E, Hall Y, Tamura MK, Chertow G. Frailty and chronic kidney disease: The third National Health and Nutrition Evaluation Survey. American Journal of Medicine. 2009; 122(7):664-71. doi: 10.1016/j.amjmed.2009.01.026

[28] Tamura MK, Larive B, Unruh M, Stokes J, Nissenson A, Mehta $\mathrm{R}$, et al. Frequent Hemodialysis Network Trial Group : Prevalence and correlates of cognitive impairment in hemodialysis patients: The Frequent Hemodialysis Network trials. Clinical Journal of American Society of Nephrology. 2010; 5(8):1429-438. doi: $10.1093 / \mathrm{ndt} / \mathrm{gfl} 275$

[29] Kurella M, Mapes DL, Port F, Chertow G. Correlates and outcomes of dementia among dialysis patients: The dialysis outcomes and practice patterns study. Nephrology Dialysis Transplantation. 2006; 21(9):2543-548. doi: 10.1093/ndt/gfl275
[30] Murray A. Cognitive impairment in the aging dialysis and chronic kidney disease populations: An occult burden. Advanced Chronic Kidney Disease. 2008; 15(2):123-32. doi: 10.1053/j. ackd.2008.01.010

[31] Kurella M, Chertow G, Fried L, Cummings S, Harris T, Simonsick E, et al. Chronic kidney disease and cognitive impairment in the elderly: The health, aging, and body composition study. Journal of the American Society of Nephrology. 2005; 16(7):2127-133. doi: $10.1681 /$ asn.2005010005

[32] Yaffe K, Ackerson L, Tamura MK, Blanc PL, Kusek J, Sehgal A, et al. Chronic renal insufficiency cohort investigators: Chronic kidney disease and cognitive function in older adults: Findings from the chronic renal insufficiency cohort cognitive study. Journal of the American Geriatrics Society. 2010; 58(2):338-45. doi: 10.1111/j.1532-5415.2009.02670.x

[33] Griva K, Stygall J, Hankins M, Davenport A, Harrison M, Newman S. Cognitive impairment and 7-year mortality in dialysis patients. American Journal of Kidney Disease. 2010; 56(4):693-703. doi: 10.1053/j.ajkd.2010.07.003

[34] Giang L, Weiner D, Agganis B, Scott T, Sorensen E, Tighiouart $\mathrm{H}$, et al. Cognitive function and dialysis adequacy: No clear relationship. American Journal of Nephrology. 2011;33(1):33-38. doi: $10.1159 / 000322611$

[35] Tamura MK, O'Hare A, McCulloch C, Johansen K. Signs and symptoms associated with earlier dialysis initiation in nursing home residents. American Journal of Kidney Disease. 2010; 56(6):1117-126. doi: 10.1053/j.ajkd.2010.08.017

[36] Berger J, Hedayati S. Renal replacement therapy in the elderly population. Clinical Journal of the American Society of Nephrology. 2012; 27(6):1039-046. doi: 10.2215/cjn.10411011

[37] Wen SS, Chan C. The elderly patient with end-stage renal disease: is dialysis the bestand only option?. Proceedings of Singapore Healthcare. 2012; 21(2):125-31. doi: 10.1177/201010581202100206

[38] Jassal S, Trpeski L, Zhu N, Fenton S, Hemmelgarn B. Changes in survival over the years 1990-1999 for elderly patients initiating dialysis. Canadian Medical Association Journal. 2007; 177(9):1033038. doi: 10.1503/cmaj.061765

[39] Lockhart T, Barth A, Zhang X, Songra R, Abdel-Rahman E, Lach J. Portable, non-invasive fall risk assessment in end stage renal disease patients on hemodialysis. Wireless Health. 2011; 2(1):84-93. doi: 10.1145/1921081.1921092

[40] Jensen J, Lundin-Olsson L, Nyberg L, Gustafson Y. Fall and injury prevention in older people living in residential care facilities. A cluster randomized trial. Annals of Internal Medicine. 2002; 136(10):733-41. doi: 10.7326/0003-4819-136-10-200205210-00008

[41] Abdel-Rahman EM, Turgut F, Turkmen K, Balogun RA. Falls in elderly hemodialysis patients. QJM: An International Journal of Medicine. 2011; 104(10):829-83. doi: 10.1093/qjmed/hcr108 